\title{
Giant pulses from the Crab pulsar
}

\section{A wide-band study}

\author{
R. Karuppusamy ${ }^{1,3}$, B. W. Stappers ${ }^{2,3}$, and W. van $\operatorname{Straten}^{4}$ \\ 1 Sterrenkunde Instituut Anton Pannenkoek, University of Amsterdam, Kruislaan 403, Amsterdam, The Netherlands \\ e-mail: ramesh. karuppusamy@gmail.com \\ 2 Jodrell Bank Centre for Astrophysics, School of Physics and Astronomy, The University of Manchester, Manchester M13 9PL, UK \\ e-mail: Ben.Stappers@manchester.ac.uk \\ 3 Stichting ASTRON, Postbus 2, 7990 AA, Dwingeloo, The Netherlands \\ 4 Centre for Astrophysics and Supercomputing, Swinburne University of Technology, Hawthorn, VIC 3122, Australia \\ e-mail: vanstraten.willem@gmail.com
}

Received 24 November 2009 / Accepted 7 February 2010

\section{ABSTRACT}

\begin{abstract}
The Crab pulsar is well-known for its anomalous giant radio pulse emission. Past studies have concentrated only on the very bright pulses or were insensitive to the faint end of the giant pulse luminosity distribution. With our new instrumentation offering a large bandwidth and high time resolution combined with the narrow radio beam of the Westerbork Synthesis Radio Telescope (WSRT), we seek to probe the weak giant pulse emission regime. The WSRT was used in a phased array mode, resolving a large fraction of the Crab nebula. The resulting pulsar signal was recorded using the PuMa II pulsar backend and then coherently dedispersed and searched for giant pulse emission. After careful flux calibration, the data were analysed to study the giant pulse properties. The analysis includes the distributions of the measured pulse widths, intensities, energies, and scattering times. The weak giant pulses are shown to form a separate part of the intensity distribution. The large number of giant pulses detected were used to analyse scattering and scintillation in giant pulses. We report for the first time the detection of giant pulse emission at both the main- and interpulse phases within a single rotation period. The rate of detection is consistent with the appearance of pulses at either pulse phase as being independent. These pulse pairs were used to examine the scintillation timescales within a single pulse period.
\end{abstract}

Key words. pulsars: individual: Crab pulsar

\section{Introduction}

Identified as the supernova remnant that resulted from SN 1054, the Crab nebula is one of the strongest radio sources in the sky, and it harbours the young neutron star PSR B0531+21. The pulsar is visible across the entire observable electromagnetic spectrum, and at radio wavelengths it is the second brightest pulsar in the northern sky. PSR B0531+21 was discovered by Staelin \& Reifenstein (1968), soon after the discovery of pulsars. This pulsar is noted for several features including the near orthogonal alignment of the magnetic and rotational axis that gives rise to the observed interpulse emission. The average emission profile of the pulsar, obtained by averaging the radio emission from many rotations of the star, exhibits a number of features that change quite remarkably with radio frequency (Moffett \& Hankins 1994). The single pulses show a large variation in amplitude and duration as a function of time. The most enigmatic of these are its occassional intense bursts known as giant pulses (Heiles et al. 1970; Staelin \& Sutton 1970). The giant pulses can be extremely narrow, of the order of 0.4 ns (Hankins \& Eilek 2007) and the pulse flux can be several 1000 times the average pulse flux. The ultrashort durations of the giant pulses imply very high equivalent brightness temperatures (Hankins et al. 2003) indicating that they originate from nonthermal, coherent emission processes. In this work, we define giant pulses as the pulses with a significantly narrower width than the average emission and contain a flux of at least 10 times the mean flux density of the pulsar.

The Crab pulsar is one of just a handful of pulsars that have been shown to have giant pulse emission. Some other pulsars, like the young Vela pulsar, also show narrow, bursty emission called giant micropulses (Johnston et al. 2001). The fluxes of these micropulses are within a factor of 3 times the average pulse flux. In the pulsars that show giant pulse emission, the pulse intensity and energy distributions exhibit power-law statistics (Argyle \& Gower 1972), while the giant micropulses give rise to log-normal distributions (Cairns et al. 2001). In contrast, the bulk of the pulsar population have pulse intensities and energies that follow either a normal or an exponential distribution (Hesse \& Wielebinski 1974; Ritchings 1976). This indicates that the giant pulses and micropulses may form a different emission population.

The Crab giant pulses have been studied by different groups, yet the nature of the emission process remains elusive. In the very early studies at low sky frequencies, the data were afflicted by dispersion smearing and scattering (Heiles et al. 1970; Gower \& Argyle 1972), but the power-law nature of the intensity distribution of giant pulses was identified. In the next major study, Lundgren et al. (1995) discuss a multi-wavelength observation of giant pulse emission, and note the possibility of a weak giant pulse emission population at radio wavelengths, which they are unable to resolve owing to insufficient sensitivity. Sallmen et al. (1999) found that the Crab giant pulses are broad band 
Table 1. Telescope parameters and observation details.

\begin{tabular}{ll}
\hline \hline Parameter & Value \\
\hline Observation duration & $21420 \mathrm{~s}$ \\
Start Epoch & $53654.726505(\mathrm{MJD})$ \\
Sky frequencies & $1311^{a}, 1330,1350,1370,1392^{a}$ \\
& $1410,1428^{a, b}, 1450 \mathrm{MHz}$ \\
Bandwidth & $8 \times 20 \mathrm{MHz}$ \\
Nominal $T_{\text {sys }}$ & $30 \mathrm{~K}$ \\
Beam size & $21^{\prime \prime} \times 1741^{\prime \prime c}$ \\
\hline
\end{tabular}

Notes. (a) These frequencies are not uniformly spaced to avoid interference.

(b) This band was not recorded due to disk failure.

(c) The beam size varies as a function of the observation time. See text for details.

at radio wavelengths. They also determine giant pulse spectral indices in the range of -2.2 to -4.9 using their widely spaced observation bands and 29 simultaneously detected giant pulses. Observations by Hankins et al. (2003) revealed that giant pulses at $5.5 \mathrm{GHz}$ contain nanosecond wide subpulses and the presence of such narrow features has been predicted in numerical modelling by Weatherall (1998). At these frequencies the radio emission character of the Crab pulsar changes, with the interpulse emission becoming dominant. A multi-wavelength radio observation of Crab giant pulses with widely spaced frequency bands $(0.43 \mathrm{GHz}$ and $8.8 \mathrm{GHz})$ is presented by Cordes et al. (2004), who discuss the effects of scintillation over a wide range of frequencies. Popov \& Stappers (2007) and Eilek et al. (2002) investigated pulse width distributions and find that narrow pulses tend to be brighter. Bhat et al. (2008) carried out a similar analysis in addition to scattering and dispersion variations in the nebula. All of these studies point to the peculiarity of the Crab pulsar and its puzzling emission process, and motivates further study in finer detail using a large number of pulses. For the work discussed in this paper, we utilised the wide band capabilities of the new pulsar machine, PuMa-II (Karuppusamy et al. 2008) and the Westerbork Synthesis Radio Telescope (WSRT) in the coherent tied-array mode. At small hour angles, the synthesised beam of the WSRT effectively resolves out the Crab nebula, reducing the nebular contribution to the system temperature. Thus the WSRT and PuMa-II combination makes this study much more sensitive in terms of signal-to-noise ratio achieved, and in number of pulses than was possible in the past. The rest of the paper is organised as follows: in Sect. 2 we describe the observational set up and data reduction, flux calibration is discussed in Sect. 3, the giant pulse characteristics are discussed in Sect. 4. We report detections of double giant pulses in Sect. 5, and the scattering analysis is presented in Sect. 6.

\section{Observations and data reduction}

The radio observations of the Crab pulsar reported here were carried out as part of a multi-wavelength observation with the Integral $\gamma$-ray telescope and the WSRT on 11 October 2005. The WSRT observations were from UTC 03.56 . 50 s. to $09^{\mathrm{h}} .36 .20^{\mathrm{s}}$. with a break of three minutes in the middle of the observation to switch data disks. The results of the $\gamma$-ray observations will be reported elsewhere.

The pulsar was observed at eight different sky frequencies in the $L$-Band, which is the most sensitive front-end receiver at the WSRT $\left(T_{\text {sys }}=30 \mathrm{~K}\right)$. The sky frequencies (see Table 1$)$ were chosen to be free of radio frequency interference. Two orthogonal polarisations of $8 \times 20 \mathrm{MHz}$ analogue signals from each telescope were 2-bit sampled at the Nyquist rate of $40 \mathrm{MHz}$. The telescope was operated in the tied-array mode in which coherent sums of the sampled voltages were formed in dedicated adder units resulting in 6-bit summed voltages. A coherent sum was achieved by determining the instrumental phase offsets between the telescopes using observations of a strong calibrator source. These phase offsets, combined with the geometrical phase offsets required for tracking the source are applied to each telescope. The resulting values were then read off as 8-bit data and recorded in the PuMa-II storage nodes. This resulted in a total of 13.5 Terabytes of raw data. After the observation, the data were processed offline using the open-source pulsar data processing software package DSPSR ${ }^{1}$. A 32-channel synthetic coherent filterbank was formed across each $20 \mathrm{MHz}$ band with coherent dedispersion applied across each of the channels using the dispersion measure (DM) of the pulsar. We obtained the DM (=56.742) from the Crab pulsar ephemeris maintained by the Jodrell Bank Observatory2 ${ }^{2}$ (Lyne et al. 1993) at the epoch closest to our observation. Frequency resolution was preserved so that studies of spectral indices, scintillation, and scattering could be carried out.

The total intensity was computed for each pulse from the dedispersed data. Giant pulses were detected by computing the peak signal-to-noise ratio (denoted by $S / N$ ). The giant pulse detection threshold was set at $S / N \geq 7 \sigma$ in each band, where $\sigma$ is the off-pulse root-mean-square noise fluctuation. Pulses below the detection threshold were discarded to ease storage requirements. The original sampling time was $25 \mathrm{~ns}$. The 32-channel filterbank and the choice of $4.1 \mu$ s final time resolution resulted in 8192 phase bins. The time resolution of $4.1 \mu \mathrm{s}$ was chosen to match the estimated scattering timescale available at the time (Sallmen et al. 1999). However, it is known from recent work by Bhat et al. (2008) that single pulses at these radio frequencies can be as narrow as $0.5 \mu \mathrm{s}$. In addition to the single pulses, average pulse profiles with 128 frequency channels in each $20 \mathrm{MHz}$ band were formed every $10 \mathrm{~s}$.

The reduced data consisted of $\sim 21000$ giant pulse candidates in each recorded band. An example candidate is shown in Fig. 1, where the pulse was detected in all bands. In the offline analysis stage, these candidates were combined in software using only pulses that show the expected dispersion delay. This method ensures that spurious signals were filtered out in our analysis. After combining in software, 12959 giant pulses were identifed to have occurred simultaneously at all observed sky frequencies. Of the 12959 pulses, 11384 were detected at the main pulse phase and 1370 at the interpulse phase of the average pulse profile.

The data were folded and the single pulses were formed using the DSPSR software package and a polynomial determined by using TEMPO (Taylor \& Weisberg 1989). The folded profiles formed in each $20-\mathrm{MHz}$ band were combined in software to validate the DM used. The combined data are shown in Fig. 2 as a frequency-phase image and shows no smearing, confirming that the value of DM is correct. A similar procedure was used to combine simultaneous giant pulses in all seven bands. Some artifacts of the 2-bit systems of the individual telescopes are visible once the profile is summed for the entire six-hour long observation. The width of these artifacts match the dispersion smearing in the bands as seen in the top panel of Fig. 2. The quantisation noise is $12 \%$ for a single telescope, whose signal is sampled

\footnotetext{
1 http://dspsr.sourceforge.net/

2 http://www.jb.man.ac.uk/ pulsar/crab.html
} 


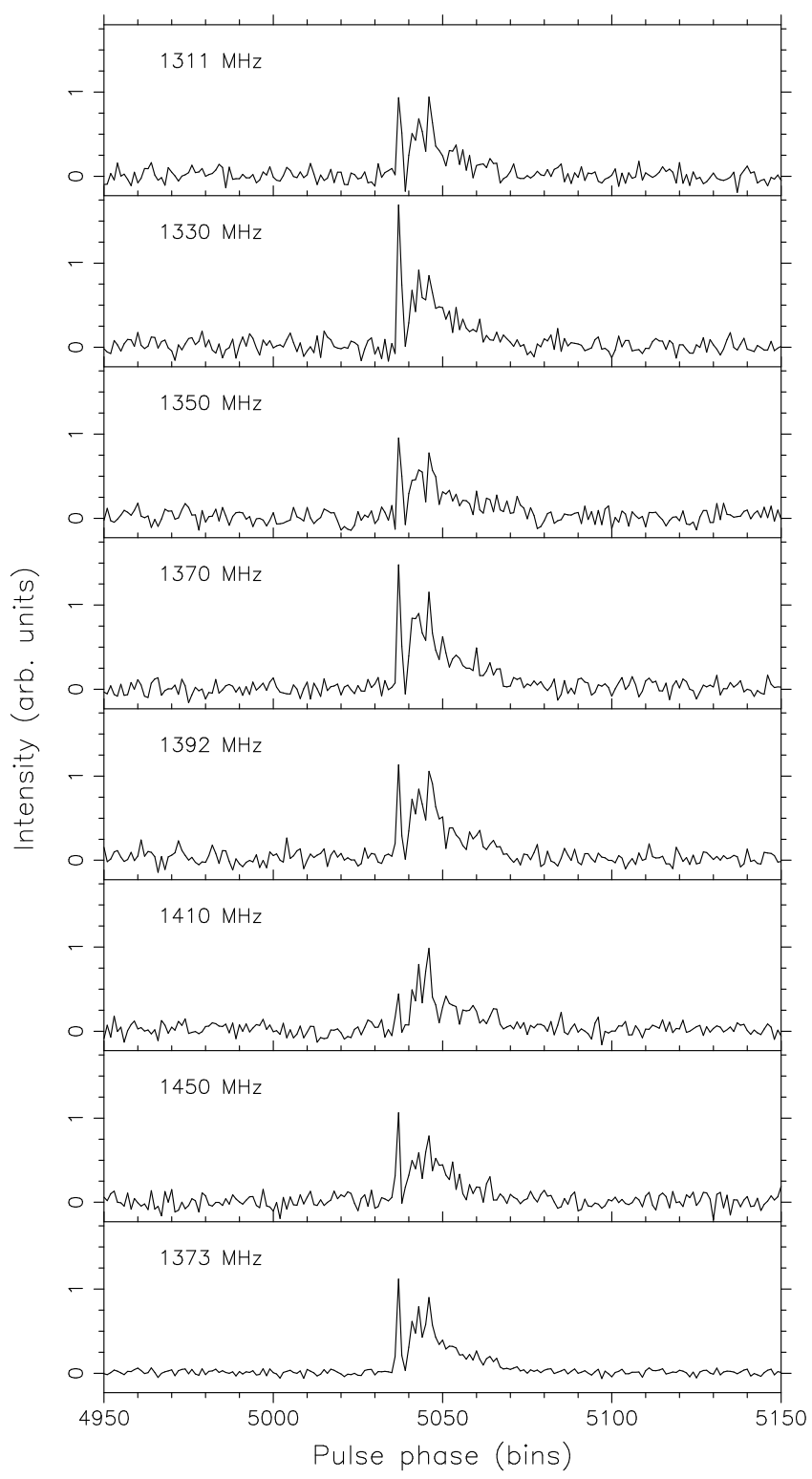

Fig. 1. Total intensity of a coherently dedispersed giant pulse at the main pulse phase detected in all recorded bands at $4.1 \mu$ s resolution. The total dispersion delay of $24.9 \mathrm{~ms}$ across the seven bands was removed for this plot. The lower most panel shows the pulse after combining the signal in all seven bands. The pulses displayed here are scaled relative to the pulse at $1330 \mathrm{MHz}$.

using 2-bits (Cooper 1970). Since signals from the 14 telescopes of the array were coherently summed, the uncorrelated quantisation noise was reduced by a factor of $\sqrt{14}$. The resulting noise of $3.7 \%$ is considered too small to be problematic in the analysis that follows. In many stages of the analysis, extensive use of the PSRCHIVE (Hotan et al. 2004) utilities was made to view and validate the pulsar data and to compute the $S / N$ used in later analysis.

\section{Flux calibration}

To establish a flux scale for the observed giant pulses, the mean system flux needs to be computed. The mean system flux is

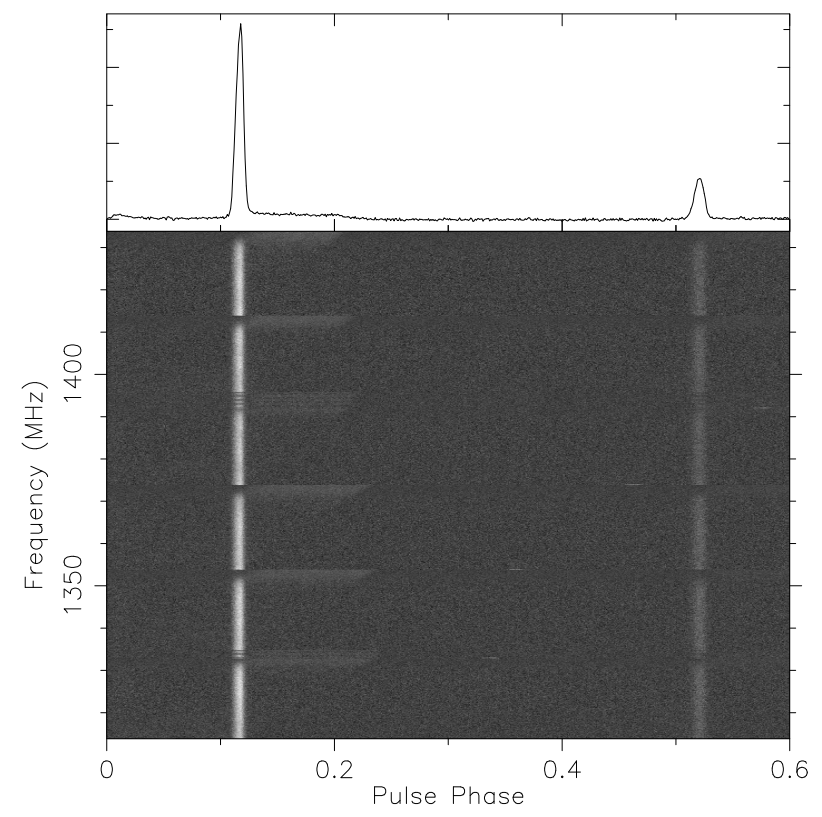

Fig. 2. The plot shows the average pulse profile (top panel) and the total intensity for six of the seven recorded bands in greyscale (lower panel). The striped nature of channels at $1330 \mathrm{MHz}$ and $1390 \mathrm{MHz}$ comes from the overlap in the adajcent frequency bands. The roll-off of the filters used in the system is also seen as a reduced intensity at the band edges. A low-level extended feature is seen at the edge (also visible in the top panel as the elevated baseline in the right side of the main pulse) of each band which is due to the 2-bit quantisation noise and is only visible in long exposures.

proportional to the rms noise variations at the telescope output and can be expressed by the radiometer equation (Dicke 1946),

$S_{\text {min }}=\frac{S_{\text {sys }}}{\sqrt{N_{\mathrm{p}} \cdot B \cdot T_{\mathrm{int}}}}$,

where, $S_{\min }$ is the rms system noise in Jy, $S_{\text {sys }}$ the total system noise, $N_{\mathrm{p}}$ the number of polarisations (=2), $B$ the bandwidth in $\mathrm{MHz}(=140)$, and $T_{\text {int }}$ the integration time in seconds. The total system noise in flux density units in Eq. (1) is the total equivalent system temperature divided by the telescope gain $\left(S_{\text {sys }}=T_{\text {total }} / G\right)$. For the WSRT, when signals from the fourteen 25-m parabolic dishes are combined, the resulting telescope gain ${ }^{3}$ is $G=1.2 \mathrm{~K} \mathrm{Jy}^{-1}$. The term $T_{\text {total }}$, can again be expressed as

$T_{\text {total }}=T_{\text {sys }}+f_{v}(t) \cdot T_{\mathrm{cn}}$.

The term $T_{\mathrm{cn}}$ is the contribution of the Crab nebula to the system temperature, while $f_{v}(t)$ is a time-dependent factor explained below. Following Bietenholz et al. (1997), we express the total flux of the Crab nebula at frequency $v$ (in $\mathrm{GHz}$ ) as $S_{\mathrm{CN}}=955 v^{-0.27} \mathrm{Jy}$, from which $T_{\mathrm{cn}}$ is computed. The WSRT is an east-west array and the coherent addition of the telescope signals results in a $21^{\prime \prime} \times 1741^{\prime \prime}$ fan beam. The Crab nebula is an extended source of size $\Omega_{\mathrm{cn}}=6^{\prime} \times 4^{\prime}$, so the WSRT's fan beam resolves the Crab nebula in the east-west direction. This in turn reduces the nebular contribution to the $T_{\text {sys }}$. However, the width of the WSRT's fan beam is not a constant, but is a function of the observation time. While the source is being tracked,

\footnotetext{
3 The telescope gain is $1.34 \mathrm{~K} \mathrm{Jy}^{-1}$ for an ideal array combiner. The reduction in gain is attributed to losses in the formation of the tied-array signal.
} 

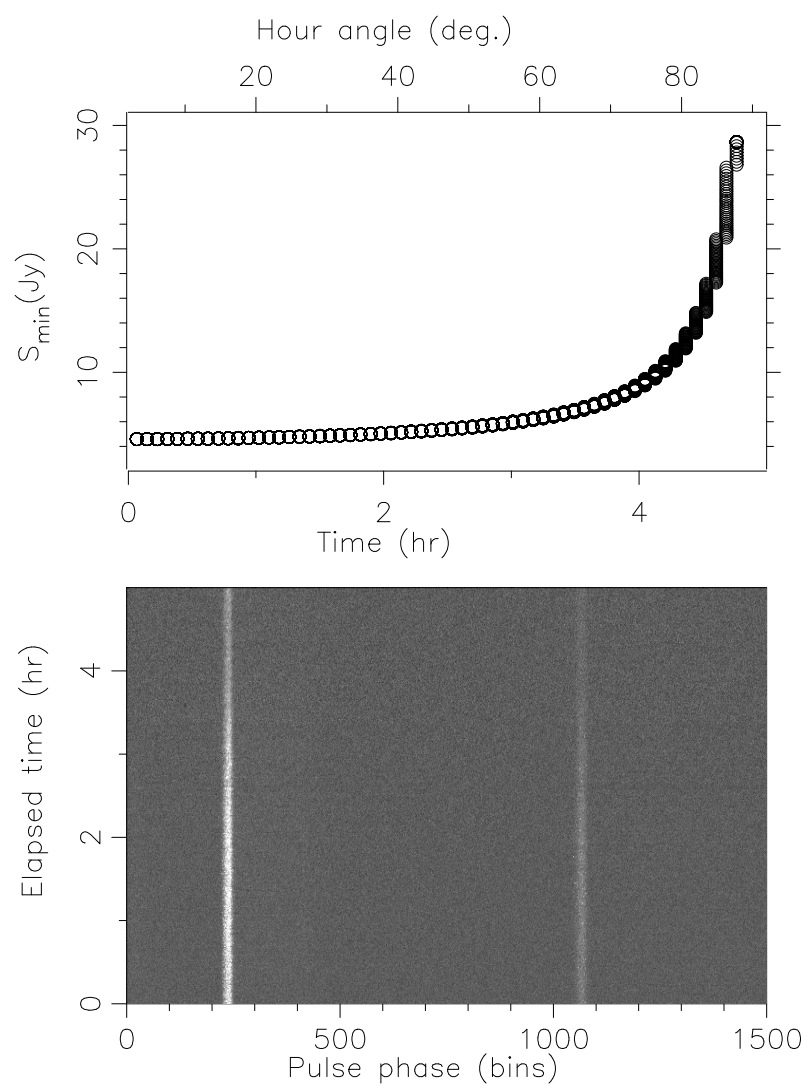

Fig. 3. The upper panel shows the change in minimum detectable signal $S_{\min }$ within a $4.1 \mu$ s time interval during the first $5 \mathrm{~h}$ of the $6-\mathrm{h} \mathrm{ob}-$ servation. The hour angle of the source is displayed on the top ordinate axis. The lower panel is the observed change in peak signal-to-noise ratio of the detected signal. The dependence of the signal-to-noise ratio on the hour angle of source is discussed in the text.

the effective width of the synthesised beam changes with hour angle (HA) and it is expressed as $\Omega_{\mathrm{A}}(t)=\Omega_{\mathrm{cn}} \cdot \lambda / D \cdot \cos (\mathrm{HA})$. In this expression HA $=t-\mathrm{RA}$, where $t$ is the local sideral time, the maximum baseline $D=2700 \mathrm{~m}$, and RA is the right ascension of the Crab pulsar. The fraction of the nebular contribution can be expressed as $f_{v}(t)=\Omega_{\mathrm{A}}(t) / \Omega_{\mathrm{cn}}$, which reaches its minimum value of 0.13 at zenith. As the source is tracked towards the horizon, the projected distance between the dishes decreases and $\Omega_{\mathrm{A}}(t)$ increases. Consequently, the observing system becomes less sensitive toward larger hour angles, or when the source rises and sets. This time dependence of the system noise is included in our flux calibration. The variation in $S_{\min }$ is shown for a bandwidth of $140 \mathrm{MHz}, N_{\mathrm{p}}=2$ and $\tau=4.1 \mu \mathrm{s}$ in the upper panel of Fig. 3. A plot of the pulse intensity during the observation (lower panel of Fig. 3) confirms this reduction in sensitivity.

The peak flux of the giant pulses were computed using the modified radiometer equation (Lorimer \& Kramer 2005) for the pulsar case, $S_{\text {peak }}=(S / N) \cdot S_{\text {min }}$. With the above considerations of the nebular contribution to $T_{\text {total }}$ and with $T_{\text {sys }}=30 \mathrm{~K}$ in the WSRT's $L$-Band, the system retained sufficiently high sensitivity in the first $15000 \mathrm{~s}$ of the observation. Two other factors have been neglected in this calibration procedure and do not contribute significantly to the $T_{\text {sys }}$ : the relative change in the orientation of the WSRT's fan beam and the Crab nebula over the course of observation and the partial shadowing of three telescopes out of the 14 for $\mathrm{HA}>54^{\circ}$ (the last $3 \mathrm{~h}$ of our observation).
Table 2. Reported sensitivity to the Crab giant pulse observations in the literature.

\begin{tabular}{lcc}
\hline \hline Reference & $\begin{array}{c}\text { Frequency } \\
(\mathrm{MHz})\end{array}$ & $\begin{array}{c}\text { Threshold } \\
(\mathrm{Jy})\end{array}$ \\
\hline Lundgren et al. (1995) & 800 & 120.0 \\
Popov \& Stappers (2007) & 1197 & $5.9^{a}$ \\
Bhat et al. (2008) & $1300 / 1470$ & $22.3^{b}$ \\
This paper & 1373 & 3.9 \\
\hline
\end{tabular}

Notes. ${ }^{(a)}$ Equivalent average pulse computed flux from the quoted $6 \sigma$ peak flux density of $142 \mathrm{Jy}$, assuming 0.036 pulse duty cycle. (b) Average pulse flux density extrapolated for $7 \sigma$ threshold, $4.1 \mu$ s time resolution and pulse duty cycle $\approx 0.036$.

\section{Single-pulse statistics}

For the analysis that follows, all pulses that were flux-calibrated as described in the previous section were used. The discussed change in system sensitivity does not limit this analysis thanks to our careful flux calibration procedure. While approximately $70 \%$ of the pulses were detected in all seven bands simultaneously, the rest were detected in two or more of the seven bands recorded. For the results described below, where applicable, only those pulses that were detected in all seven bands were used and explicitly mentioned.

\subsection{Pulse intensity distributions}

The giant pulse fluxes of the Crab pulsar contribute to the long exponential tail of the single pulse intensity histograms (Argyle \& Gower 1972), while the normal pulsars show Gaussian or exponential pulse intensity distributions (Hesse \& Wielebinski 1974). Figure 4 shows the average pulse flux distribution for pulses detected in at least two of the seven recorded bands. The average pulse flux is computed by integrating all emission within the equivalent width, $W_{\text {eq }}$ of the giant pulse (see Sect. 4.4). This value is averaged over the pulse period to obtain the average pulse flux. The pulse in each band was detected based on a threshold of $7 \sigma$. A pulse detected in two bands satisfies the $\sqrt{2} \times 7=9.89 \sigma$ limit. In the first three hours of the observation (when the system was most sensitive), the flux equivalent system noise in $4.1 \mu \mathrm{s}$ is $109 \mathrm{Jy}$. Averaged over the pulse period, a pulse of $S / N=9.89 \sigma$ corresponds to an average pulse flux density of $3.9 \mathrm{Jy}$. This implies that it is sensitive to all pulses greater than $27 \times\langle F\rangle$, where $\langle F\rangle=14 \mathrm{mJy}$ is the average flux density of the Crab pulsar. Therefore, the flux distribution computed here contains a good fraction of weak giant pulses compared to those reported elsewhere (see Table 2).

The intensity distributions displayed in Fig. 4 shows at least two components: a peak at or below $\sim 4 \mathrm{Jy}-$ the weak pulses that may comprise the trailing part of the normal pulse distribution. The next component peaking at $\sim 20 \mathrm{Jy}$ resembles a lognormal distribution with a power-law tail. The bright giant pulses result in the extended power-law tail and is described by $N_{F} \propto F^{\alpha}$, where $N_{F}$ is the number of pulses detected in $1.8 \mathrm{Jy}$ flux intervals of $F$. The value of $\alpha=-2.79 \pm 0.01$ and $\alpha=-3.06 \pm 0.06$ was determined from the best fits to the data in the interval $118 \mathrm{Jy} \leq$ $F \leq 2000 \mathrm{Jy}$ and $40 \mathrm{Jy} \leq F \leq 596$ Jy for the giant pulses in the main- and interpulse, respectively. Visual inspection of Fig. 4 shows that the distribution is multi-modal, with giant pulses in the region $F \gtrsim 10 \mathrm{Jy}$ and the pulses below this limit possibly representing normal pulses. 

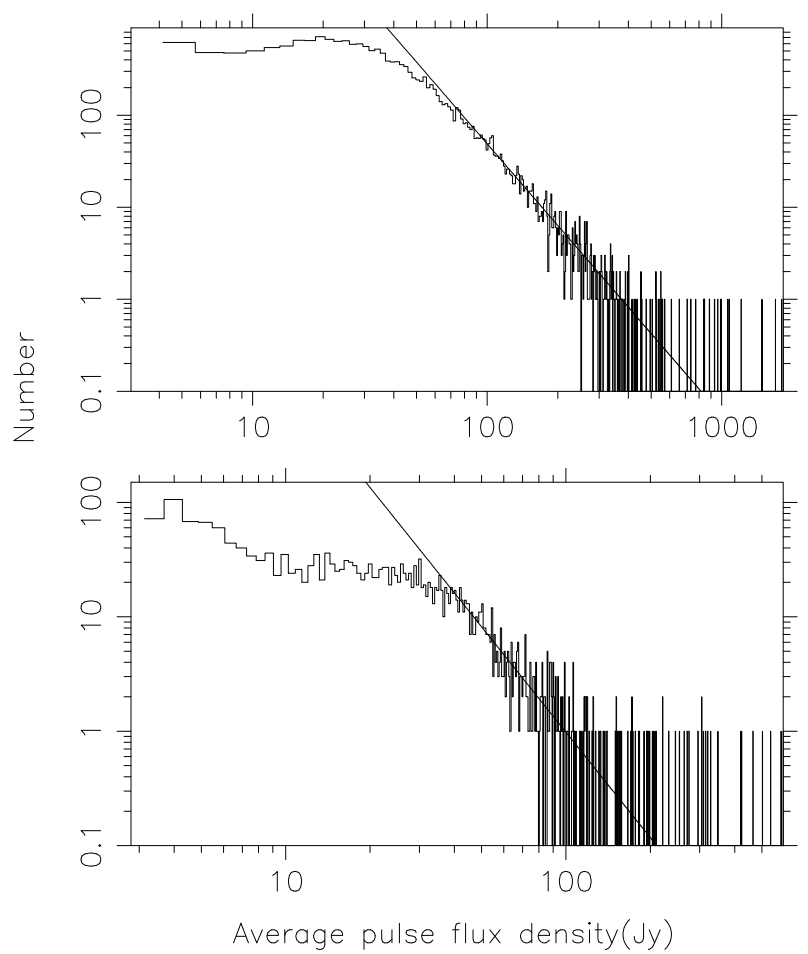

Fig. 4. Distribution of the pulse intensity of all giant pulses detected at the main- and interpulse phases in the upper and lower panels, respectively. The long tail results from the giant pulse emission. The best fit power-law curve is shown with slope $-2.79 \pm 0.01$ for the pulses in main pulse phase and $-3.06 \pm 0.06$ for the pulses in the interpulse phase. Both distributions show an excess near $4 \mathrm{Jy}$ and come from the rounding off in $W_{\text {eq. }}$. (see text for details).

It is worth noting the differences in the intensity distributions displayed in Fig. 4. While the distribution of the giant pulses in the main pulse phase shows a clear turn over at $\sim 20 \mathrm{Jy}$, the emergence of a bimodality in the region containing weak pulses is evident in the intensity distribution of the interpulse giants. The distribution corresponding to the interpulse phase also shows a flattening in the 10-30 Jy region. The clear excess of weak pulses in both the distributions in the region $F \leq 4 \mathrm{Jy}$ is due to our method of setting $W_{\mathrm{eq}}=4.1 \mu$ s (equal to the time resolution). In this case the emission window we considered is dominated by noise or weak and narrow pulses. The slopes of the power-law models obtained here can be compared to the values reported earlier. Figure 4 of Lundgren et al. (1995) shows a slope of $-3.46 \pm 0.04$ for data at $800 \mathrm{MHz}$, which is slightly steeper than the slopes of the main- and interpulse distributions derived here. Cordes et al. (2004) derive a value of $\sim-2.3$ at $433 \mathrm{MHz}$ and Bhat et al. (2008) found $-2.33 \pm 0.14$ at $1300 \mathrm{MHz}$, which are comparable to the slope the main pulse intensity distribution in our work. The slopes of the intensity distribution reported here generally agree considering the effect of low number statisics and/or dispersion smearing in the observations reported elsewhere. While this experiment was sensitive to much lower fluxes, the long observation time has also enabled the detection of rarer bright pulses.

\subsection{Pulse energy distributions}

The relative occurrence rates of giant pulses is displayed as a cumulative probablity distribution of the individual pulse energies in Fig. 5. The pulse energy is computed by multiplying the

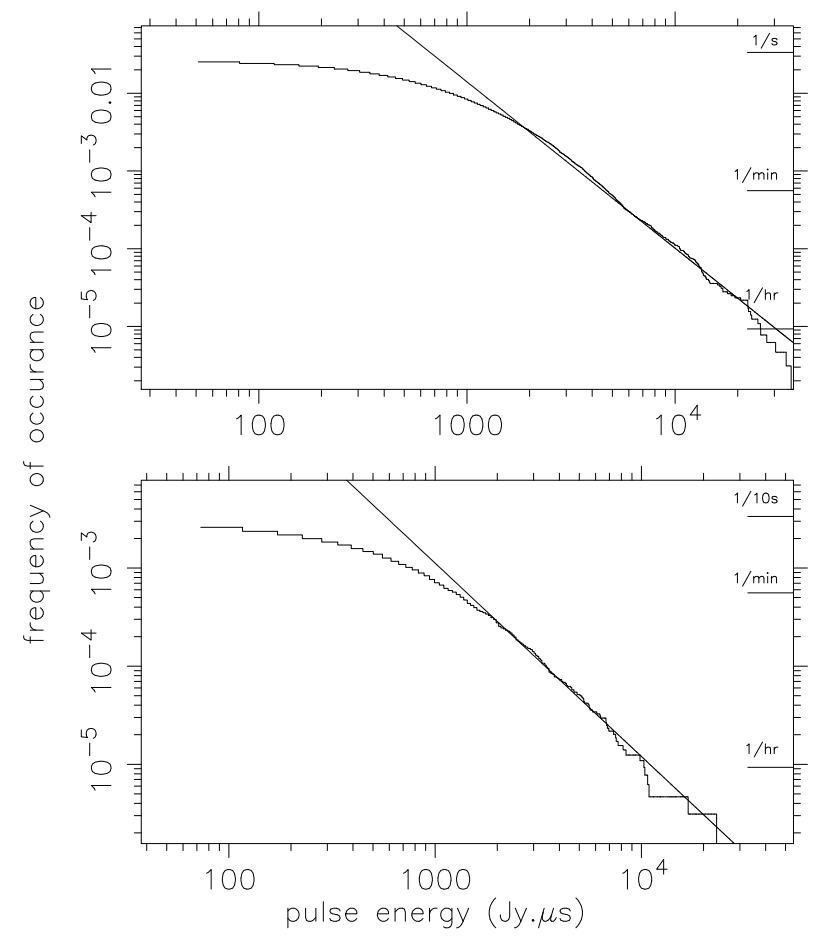

Fig. 5. The cumulative probablity distribution of the energy in giant pulses detected at the main pulse and the interpulse phases in the upper and lower panels, respectively. The $y$-axis is the fraction of the total number of pulses and pulse energy is plotted on the $x$-axis. Also shown are the occurrence rates per minute, second and hour.

equivalent width, $W_{\text {eq }}$, and the average pulse flux. As described in Sect. 4.1, we computed the best fits to the cumulative probablity distributions of the main- and interpulse giants. The powerlaw curve with $\alpha=-2.13 \pm 0.007$ and $\alpha=-1.97 \pm 0.006$ fits the data for pulse energies at the main- and inter pulse phases, respectively. The break seen at $\sim 2000 \mathrm{Jy} \mu$ s is consistent with the break value reported by Popov \& Stappers (2007). The emission at the interpulse phase shows a somewhat shallower power-law.

It is known from Popov \& Stappers (2007) that the powerlaw index has a width dependence, varying from -1.7 to -3.2 as the pulse width increases. Based on this variation, the index we find is in good agreement with Popov \& Stappers (2007) and Bhat et al. (2008) $(-1.88 \pm 0.02$ at $1300 \mathrm{MHz})$. However, we fit only a single power law unlike the two power-law fits found by these authors. Partial fits to the low-energy pulses yield more than two components, with shallower power-law indices indicating a simple dual-component fit is insufficient. One explanation for this can be the bias introduced by setting $W_{\mathrm{eq}}=4.1 \mu \mathrm{s}$ for narrow pulses, overestimating the pulse energy. However, this can only be a minor contribution and is an argument that there is a clear break in the intensity distribution. To compare the occurrence rates we see here, we proceed to derive the rates from the arrival times of the giant pulses in the next section.

\subsection{Giant pulse rates}

The distribution of the separation times between successive giant pulses is plotted in Fig. 6. If the giant pulses are mutually exclusive events independent of each other, then the arrival time separation follows a Poisson process (Lundgren et al. 1995). The probablity of a giant pulse occurring in the interval $x$ is then given by $P(x)=\mu x \cdot \mathrm{e}^{-\mu x}$, where $\mu$ is the mean pulse rate. Since 


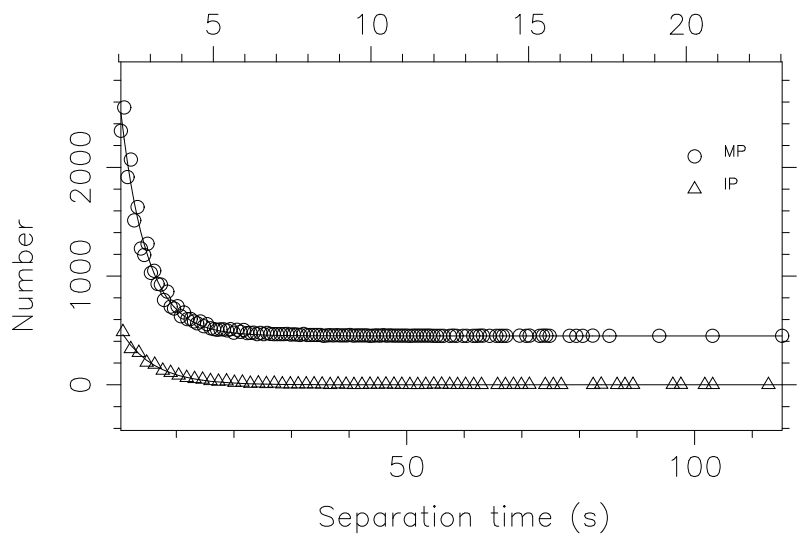

Fig. 6. The symbols show the distribution of separation times between successive giant pulses at the main- and interpulse phases and the solid lines are the best fits to the distribution. The top ordinate axis corresponds to the curve and data for the pulses at the main pulse phase and are offset by 450 for clarity.

our data only consist of giant pulses, we expected to see an exponential reduction in the separation time between the pulses. Figure 6 shows the fits to the separation times at both the interand main-pulse phases.

Functions with an exponential decay with time constants $1 / \tau=1.1 \pm 0.02$ and $1 / \tau=0.172 \pm 0.003$ are in excellent agreement with the data at the main- and interpulse phases, respectively. From the values of $\tau$, the mean giant pulse rates are one main- pulse giant every $0.9 \mathrm{~s}$ and one inter pulse giant every $5.81 \mathrm{~s}$ observed above our threshold limit of $3.9 \mathrm{Jy}$. At these frequencies, the interpulse giants are comparatively less numerous as is evident from our data. For comparision, the inter-pulse giants are brighter and more frequent in frequency bands above $5.5 \mathrm{GHz}$ (Cordes et al. 2004). The combined rate of the giant pulses (fit and data not shown) is one pulse every $0.803 \mathrm{~s}$. The foregoing discussion confirms earlier predictions that the giant pulse rate increases with frequency for the Crab pulsar (Lundgren et al. 1995; Sallmen et al. 1999). The effect of the WSRT's sensitivity reduction towards the end of the observation, as displayed in Fig. 3, may have contributed to the long tail of the distribution, where fewer pulses were detected than in the first half of the observation. However, the rate derived here is robust, since the system had sufficiently high sensitivity in the first half of the observation.

\subsection{Width distributions}

The equivalent pulse width, $W_{\text {eq }}$ is defined as the width of a tophat pulse with height equal to the peak intensity of the pulse. $W_{\mathrm{eq}}$ for the giant pulses detected in all seven bands was computed. The results are displayed in panels on the right in Fig. 7. We express $W_{\text {eq }}$ as

$W_{\mathrm{eq}}=\frac{1}{I_{\max }} \times \sum_{i=n_{1}}^{n_{2}} I_{i} \times 4.1 \mu \mathrm{s}$,

where $I_{\max }$ is the peak intensity, $I_{i}$ the intensity in the pulse emission window defined by bins $i=n_{1} \ldots n_{2}$ and is equal to $1 \mathrm{~ms}$ in our case. Thus $W_{\text {eq }}$ can be viewed as the equivalent width of a rectangular pulse in $\mu$ s that has the same area as the giant pulse, with height $I_{\max }$.

The giant pulses at these frequencies can be quite narrow. For instance, Bhat et al. (2008) find pulse widths to be $0.5 \mu$ s and

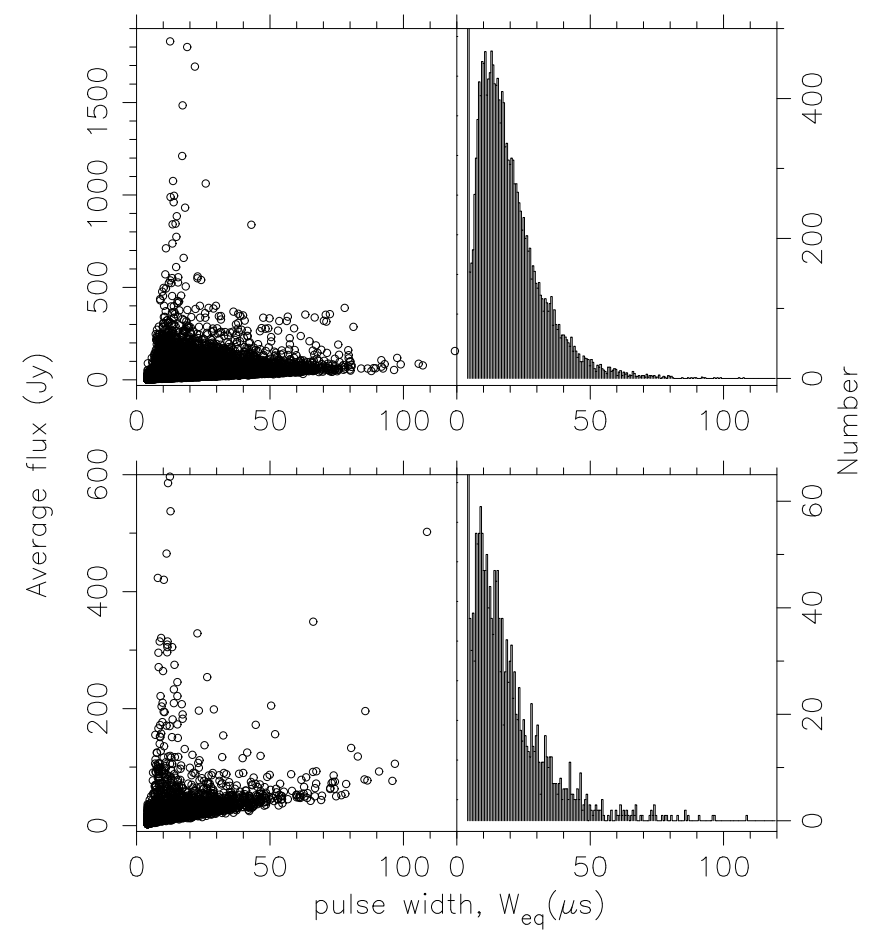

Fig. 7. Plot of intensity against pulse width for the main- and interpulse windows in the top left and lower left panels. Histograms of equivalent pulse widths are shown in the top right and lower right panels. The distribution has an exponential envelope. For pulses with computed $W_{\mathrm{eq}}<4.1 \mu \mathrm{s}$ due to random noise fluctuations the widths were rounded off to $4.1 \mu \mathrm{s}$.

Eilek et al. (2002) found $0.2 \mu \mathrm{s}$. Our method of data reduction allowed a time resolution of $4.1 \mu \mathrm{s}$, so pulses with $W_{\mathrm{eq}}<4.1 \mu \mathrm{s}$ were taken to have a width equal to $4.1 \mu \mathrm{s}$. This results in some pulses being underestimated in flux and overestimated in equivalent width. The computed equivalent widths range from $4.1 \mu \mathrm{s}$ to $\sim 120 \mu \mathrm{s}$, and we find that bright pulses tend to be narrow as seen in the left hand panels of Fig. 7. This was also suggested by Sallmen et al. (1999) and shown by Eilek et al. (2002). Popov \& Stappers (2007) found a similar behaviour in addition to a width-dependent break in the power-law fits to the pulse-energy distribution.

In the seven closely spaced radio bands observed, we note that a vast majority of the pulses have widths larger than $4.1 \mu \mathrm{s}$. This is seen in the pulse width histograms at the two pulse phases, displayed in the panels on the right in Fig. 7. The distribution shows a peak at $\sim 16 \mu \mathrm{s}$, which is 4 times our ultimate time resolution in the main pulse, and the peak shifts towards narrower timescales for the interpulses. We find less than $9 \%$ of the pulses with $W_{\mathrm{eq}}=4.1 \mu \mathrm{s}$, indicating that the majority of the pulses show wider widths than our time resolution. The shape of the width distribution is similar at both the main- and interpulse phases. The contribution to the tail region of the distribution comes from scatter broadened pulses.

\subsection{Spectral index of giant pulses}

The data were recorded in 7 different radio bands each $20 \mathrm{MHz}$ wide in the frequency range $1300-1450 \mathrm{MHz}$, and several thousands of pulses were detected simultaneously in all bands. The spectral index of individual pulses was computed by modelling the flux variation of a giant pulse as $S(v) \propto v^{k}$. Here, $S(v)$ is the flux of the giant pulse at frequency $v$, and $k$ the spectral index. 


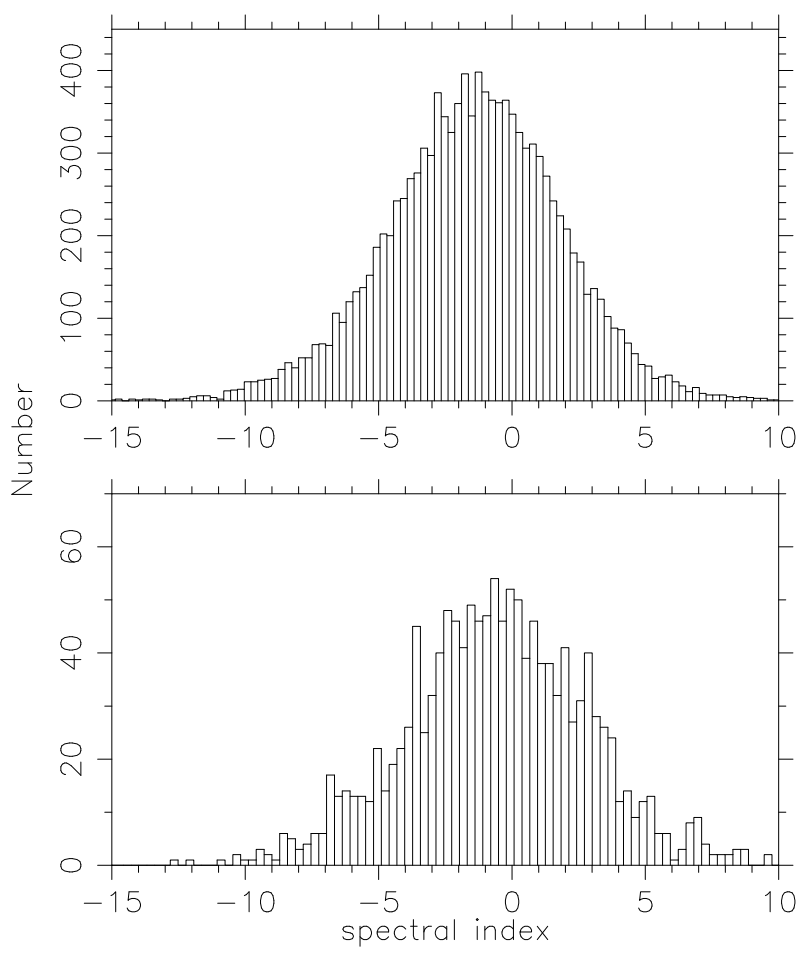

Fig. 8. Histogram of spectral indices for the giant pulses detected at the main pulse (bottom panel) and the interpulse phase (top panel). The spread in the distributions is indicative of fitting errors. See text for details.

The histograms of the derived spectral indices are displayed in Fig. 8 for the giants at both pulse phases. A large dispersion in the spectral index is seen, with values $-1.44 \pm 3.3$ for the mainand $-0.6 \pm 3.5$ for the interpulse giants.

These spectral index values are quite a bit shallower than those detected previously (see Introduction) over wider frequency separations. We therefore consider the effects of diffractive interstellar scintillation (DISS) on the spectral index estimates. Strong DISS results in pulse intensity variations within each of the seven bands. The effect of scintillation is to modulate the observed pulsar signal in both time and frequency. This is seen as regions of enhanced or diminished brightness in a grey scale plot of the intensity as a function of time and frequency. These regions are known as scintles. We estimate the scintillation bandwidth based on the pulse scatter timescales, $\tau_{\mathrm{s}}=395 \pm 50 \mu \mathrm{s}$ at sky frequency of $200 \mathrm{MHz}$, as reported in the work of Bhat et al. (2007). We further make use of their revised $\tau_{\mathrm{s}} \propto v^{-3.5}$ frequency scaling and consider that the scintillation bandwidth and scattering timescale are related by $2 \pi \Delta v_{\mathrm{d}} \tau_{\mathrm{s}}=C_{1}$, where the constant $C_{1}=1.05$ for a thin scattering screen (Cordes et al. 2004). From these considerations $\Delta v_{\mathrm{d}} \approx 0.25-0.38 \mathrm{MHz}$ in the $1300-1460 \mathrm{MHz}$ band. On examining a few giant pulses by eye, it was clear that some of the scintles are resolved, while some were narrower than our channel width of $\Delta v=0.625 \mathrm{MHz}$. Thus, in the flux obtained by integrating the signal in the $20 \mathrm{MHz}$-wide bands, the scintles tend to average out. This implies that scintillation does not cause the spread in the individual giant-pulse spectral indices. Moreover, with such narrow scintillation bandwidths, averaging over many giant pulse spectral index determinations as we have done here would give an average spectral index that reflects the true average spectral index.
Refractive interstellar scintillation (RISS) cannot corrugate the spectra of single pulses, since the pulse intensity variations due to RISS are noticeable in observation of the order of a few days (Lundgren et al. 1995). However, the pulses do have a significant structure that is intrinsic to the emission process. One example is displayed in Fig. 1 and these pulses do contribute to the spread in the computed spectral indices. In this figure, it is clear that the leading short burst shows considerable variation across the seven bands, while the scattered trailing part of the pulse is correlated across frequency. This is again similar to what Hankins \& Eilek (2007) find, as shown in their Fig. 4, but at a much higher frequency of $\sim 9 \mathrm{GHz}$.

Sallmen et al. (1999) find that the spectral index variation is between -4.9 and -2.2 based on 29 pulses they observed in two bands centred at $1.4 \mathrm{GHz}$ and $0.6 \mathrm{GHz}$. The spread in the indices computed here and that of Sallmen et al. (1999) points to the stochastic nature of the giant pulse emission process and/or the disturbed plasma flow in the magnetosphere caused by strong plasma turbulence (Hankins \& Eilek 2007). The giant pulses used in this analysis were detected in all seven bands and represent $70 \%$ of all detected pulses in our data. Since each of our bands is $20 \mathrm{MHz}$ wide, detection in seven bands implies an emission bandwidth of at least $\Delta v=140 \mathrm{MHz}$. This suggests that the emission bandwidth of Crab giant pulses is potentially greater than $\Delta v / v=0.1$, unlike the giant pulse emission from the millisecond pulsar B1937+21 (Popov \& Stappers 2003). We note that the $\Delta v / v=0.8$ for the Crab giant pulses reported by Sallmen et al. (1999) was based on 29 simultaneous giant pulses from their 90-min observation ( $\sim 161086$ stellar rotations). Those 29 pulses could have been chance detections, while the $\Delta v / v=0.1$ limit derived here comes from a much larger sample of giant pulses so is more robust. We detected a total of 17587 giant pulses, of which approximately 4000 were detected in less than 7 bands. Clearly it is impossible to include the pulses detected in only a few bands in this analysis as that would increase the dispersion in the spectral indices computed; however, this lack of detection in all bands, for pulses which were clearly detected in the other bands, is an argument for there being some narrow band effects that appear to modulate the giant pulse intensity.

\section{Double giant pulses}

During direct inspection of some giant pulses, it was noticed that occasional giant pulse emission was evident at both the mainand interpulse phases within a single rotation period of the star. To determine how many such pulses were present, the following search algorithm was used. First, the giant pulses detected in all seven bands were combined in software across the frequency bands. The pulses were then averaged over polarisation and frequency to create single pulse total intensity profiles. The search algorithm was made sensitive to emission at both emission windows (main- and interpulse) by traversing each pulse profile twice; in the first pass, the emission peak and phase information was recorded, following which a search is made in the other emission window i.e. if a pulse was detected at the main pulse phase we check whether a pulse is also seen at the interpulse phase. All pulses that show signal $\geq 5 \sigma$ in the second emission window are collected separately. The pulses returned by the search procedure were examined by eye to validate the double pulse nature. To our knowledge, this is the first instance of this phenomena being reported. A total of 197 pulses that show emission at both pulse phases were found in our data set above the $5 \sigma$ detection threshold. 


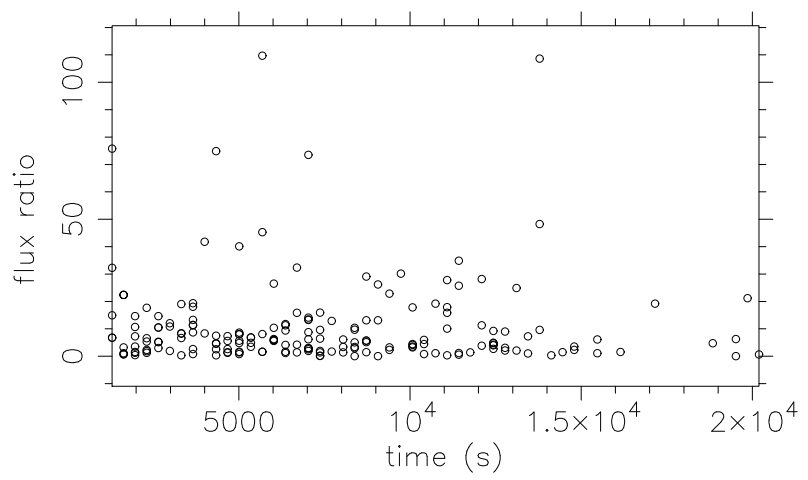

Fig. 9. Detected double giant pulses shown as a ratio of the main pulse to the interpulse flux. The $x$-axis shows time since the start of the observation.

To consider how likely this is to happen by chance, we note that the observation lasted 643263 rotations of the star and 11584 and 1375 giant pulses were found at the main- and interpulse phases, respectively, above the $7 \sigma$ detection threshold in each band. Since these giant pulses were detected in all seven bands, the effective threshold is now $\sqrt{7} \times 7 \sigma=18 \sigma$. If the $18 \sigma$ criterion is used to search for the double pulses, a total of 17 pulses are seen. In other words, only 17 pulses in the 197 detected show $S / N \geq 18 \sigma$ in either of the two emission windows. Let the giant pulses occurring at the two pulse phases be independent events, with individual probablitites $P(A)$ and $P(B)$. The chance of two giant pulses occurring within a single rotation period is the joint probablity $P(A, B)=P(A) \cdot P(B)$. Thus the chance of detecting a giant pulse above the $18 \sigma$ threshold limit at the main- and interpulse phases are $P(A)=11584 / 643263$ and $P(B)=1375 / 642263$ leading to $P(A, B)=3.5 \times 10^{-5}$. We therefore expect a total of $P(A, B) \times 643263=24$ pulse periods with pulses at both phases in our data. The detection of 17 pulses is thus consistent with the expected 24 pulses.

As seen above, combining the seven bands improves sensitivity and allows the detection of weaker pulses. Considering pulses with $S / N$ greater than $5 \sigma$ in the second emission window resulted in the detection of an additional 180 double pulses. While the 197 pulses detected are not sufficient to perform meaningful statistics of these pulses, in Sect. 6.1 we use our population of double giant pulses to study scintillation and scattering within a 0.5 rotation of the pulsar.

Although the appearance of the pulses in the same rotation period is consistent with the individual occurrence rates, we compared the GP properties at each phase. In the double pulses, the emission in the interpulse phase is typically narrower ( $W_{\text {eq }} \lesssim 16 \mu \mathrm{s}$ ) than the emission at the main pulse phase and pulses at the main pulse phase are typically brighter, as shown in Fig. 9. In both cases this is consistent with the known population of GPs at each phase. A similar analysis to the one in Sect. 4.3 was done to determine the rate of double pulses and a rate of 1 pulse in $84 \mathrm{~s}$, or one in 2545 rotations of the star was found to have giant pulse emission at both pulse phases. Thus, given the narrowness and very low occurrence rates of these pulses, they were easily missed in earlier observations.

\section{Single-pulse scattering}

The frequency resolution and large bandwidth of our data benefits scattering and scintillation checks on the individual pulses in two ways. First, the pulses detected in 7 bands are combined in software to give 224 channels across the $140 \mathrm{MHz}$ bandwidth allowing examination of scintillation. Second, the large bandwidth of the combined pulse increases sensitivity and makes it possible to identify low-level extended scatter tails. To characterise the scattering time $\tau_{\mathrm{s}}$ in the pulsar signal, we computed the extent of pulse broadening in the individual giant pulses. If the pulses are scattered by a thin-screen between the source and the observer, the pulses can then be modelled as an one-sided exponential with a vertical rise and a rapid decay (Williamson 1972). This can be written as

$f(t)= \begin{cases}\mathrm{e}^{-t / \tau_{\mathrm{s}}} & \text { if } t \geq 0 \\ 0 & \text { if } t<0 .\end{cases}$

This model was fit to the data using a least-squares minimisation and the $1 / e$ time derived from the models was taken as $\tau_{\mathrm{s}}$ of an individual giant pulse. It is known from the work of Sallmen et al. (1999), that a single one-sided exponential is not sufficient to model the complex structure of the giant pulses at this frequency. However, The large majority of pulses in our data show that the single exponential model agrees within $10 \%$ error. Therefore, we proceeded with the single exponential fits. The values of $\tau_{\mathrm{s}}$ as a function of observing time and their distribution are shown in the upper and lower panels of Fig. 10, respectively. The reduction in the scattering time towards the end of the observation is consistent with scattered pulses tending to be dimmer, hence below the detection threshold. Only sufficiently bright pulses are detected in the sensitivity limited part of the observation, as discussed in Sect. 3. The scatter tail is also not discernible from the system noise in this part of the observation, limiting the determination of $\tau_{\mathrm{s}}$. However, there were fewer pulses so they did not contribute to the distribution of $\tau_{\mathrm{s}}$ (lower panel of Fig. 10) significantly.

The lower panel of Fig. 10 shows an exponential envelope in the distribution of $\tau_{\mathrm{s}}$. The individual pulse scattering time varies from $4.1 \mu \mathrm{s}$ to $\sim 90 \mu \mathrm{s}$. The large number of pulses in the distribution with $\tau_{\mathrm{s}} \approx 4 \mu \mathrm{s}$ is related to our ultimate time resolution of $4.1 \mu \mathrm{s}$. This also implies that a large fraction of the pulses have scattering time $\tau_{\mathrm{s}} \leq 4.1 \mu \mathrm{s}$. At a slightly earlier epoch than our observations, Bhat et al. (2007) determined a value of $\tau_{\mathrm{s}}=395 \pm 50 \mu \mathrm{s}$ at $200 \mathrm{MHz}$. Using their revised frequency scaling of $\tau_{\mathrm{s}} \propto v^{-3.5 \pm 0.2}$, the scattering time at the centre of our band $(1373 \mathrm{MHz})$ is $0.47 \pm 0.05 \mu \mathrm{s}$. At a slightly later epoch, Bhat et al. (2008) find a value of $\tau_{\mathrm{s}}=0.8 \pm 0.4 \mu$ s at $1300 \mathrm{MHz}$, which contrasts with the value of $8 \mathrm{~ms}$ at $111 \mathrm{MHz}$ (or $1.4 \mu \mathrm{s}$ at $1300 \mathrm{MHz}$ using a $v^{-3.5}$ scaling law) reported by Kuzmin et al. (2008). With our data, we are not sensitive to scatter times below $4.1 \mu \mathrm{s}$, but to the dispersion seen in the histogram of scatter times in Fig. 10 shows that variations can even be expected within a single observation of six hours. We again refer to Fig. 1 for an example of the extreme form of this variation: the different parts of the same pulse show different scattering effects, imparting a significant structure to the pulse. In their work on DISS, Cordes \& Rickett (1998) emphasise that considering the $1 / e$ time equal to $\tau_{\mathrm{s}}$ is only valid for a thin screen and does not always hold. In light of the limited validity in interpreting the $1 / e$ time and the spread in the values of scatter times found in our analysis, we suggest that the scattering in the direction of Crab pulsar cannot be modelled by single thin screen. The spread in $\tau_{\mathrm{s}}$ ranges from $\leq 4.1 \mu$ s to $\sim 120 \mu$ s in our $\sim 6$ hour-observation. This proves most of the scattering cannot be due to the ISM, as the line of sight through the ISM does not change rapidly enough to explain these variations. Therefore, the bulk of scattering should orginate in the Crab nebula. The nebula can clearly give rise to a complex screen or changes in the structures in the vicinity of 

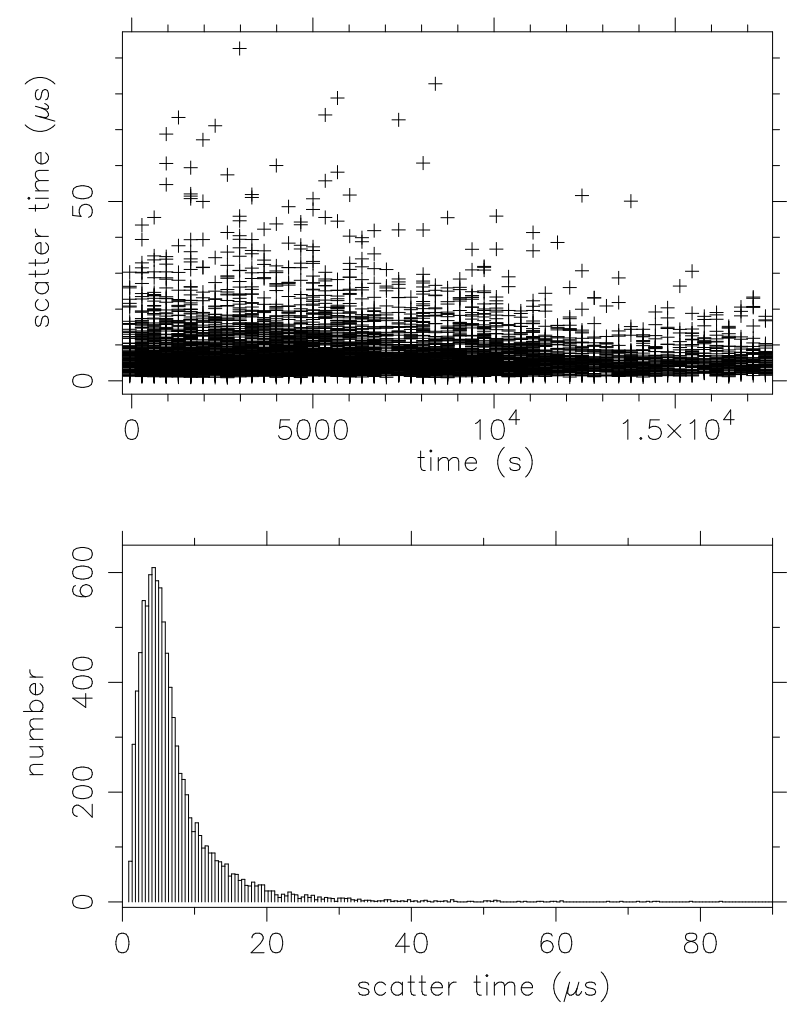

Fig. 10. Upper panel: a plot of the values of time constant $\tau_{\mathrm{s}}$ from the fits to the scattering in the entire duration of observation for those pulses detected at the main pulse phase. The lower panel displays a histogram of the time constants obtained from exponential fits to the scatter tails of individual pulses. The values of $\tau_{\mathrm{s}} \leq 4.1 \mu \mathrm{s}$ are from pulses narrower than our time resolution and those that are likely to be free of scattering.

the pulsar that give rise to the short-term changes in scattering time (Backer et al. 2000; Lyne et al. 2001; Sallmen et al. 1999). The scattering of pulses cannot be in the pulsar magnetosphere. In that case the pulses at lower frequencies that originate higher up in the magnetosphere should show lower scatter times, because according to the standard pulsar models, the number density of charged particles is lower in the upper magnetosphere (Lyubarskii \& Petrova 1998). However, $\tau_{\mathrm{s}}$ scales with frequency as $v^{-3.5}$ (Popov et al. 2006), and this does not support the hypothesis that scattering could have its orgins in the pulsar magnetosphere.

The diffractive scintillation timescale, $\Delta t_{\text {DISS }}$ at this frequency was estimated by Cordes et al. (2004) as $25.5 \mathrm{~s}$, based on pairs of single pulses with sufficient $S / N$. However, the pulse pairs they used were separated in time by a few pulse periods. Since our data has good frequency resolution (224 frequency channels across $140 \mathrm{MHz}$ ), and we detected several pulses with multiple components, we proceeded to estimate possible variations in the scintillation time on shorter timescales.

\subsection{Scintillation within single pulses}

The scintillation timescale within single pulses was estimated using those pulses that show well separated components and the double pulses discussed in Sect. 5. The search for at least two components in single pulses was carried out based on the component separation of $\sim 25 \mu \mathrm{s}$. This was done by examining the pulses by eye, after an automated first pass. The first pass provided 451 giant pulse candidates, 368 of those displayed at least two distinct shots in the main pulse phase, and 18 candidates

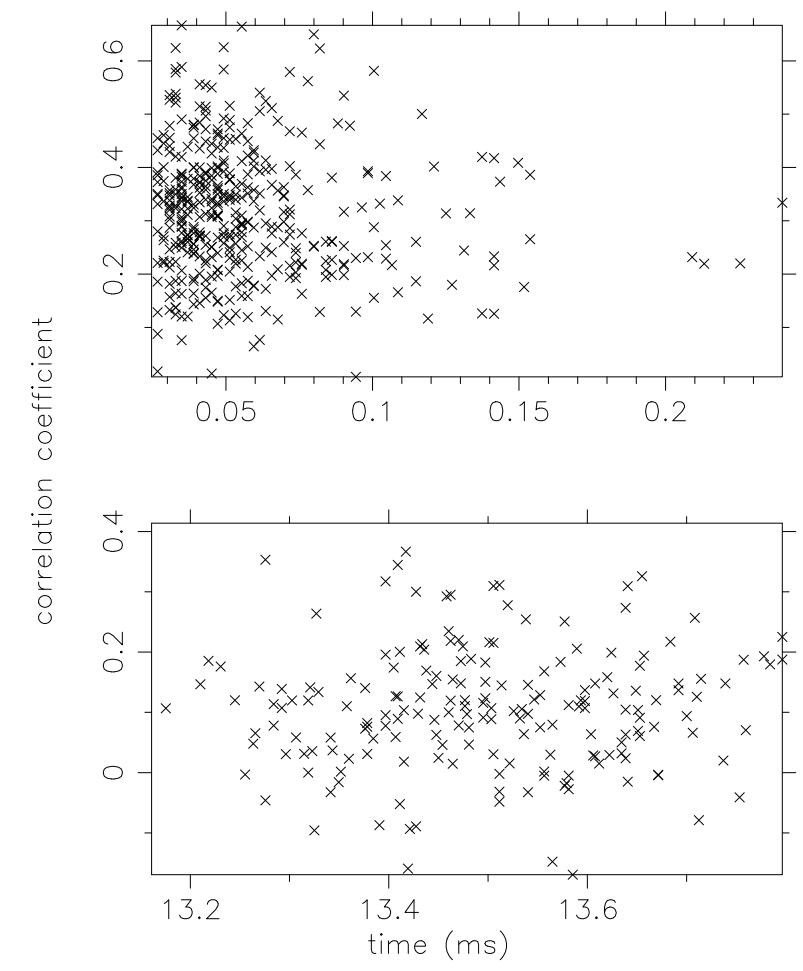

Fig. 11. Correlation coefficients of the spectra within a single pulse period. Top panel shows correlation between the two components of giant pulse, while lower panel is the double giants. The separation between the components $\tau$ is shown in the abscissa.

were found in the interpulse phase. The 197 double pulses were included in this analysis. Assuming that the two shots of pulses are intrinsic to the pulsar emission and that the scattering screen remains stable within a pulse period, any scintillation would affect the two components similarly, introducing a correlated frequency structure. The scintillation timescale is then the $1 / e$ point along the time axis of the 2-dimensional intensity correlation function, $C(\delta v, \tau)=\langle I(t, v) . I(t+\tau, v+\delta v)\rangle$ of the spectrum (Cordes 1986). The computed correlation coefficients between the two components and the double pulses are displayed in Fig. 11.

The correlation coefficient of $\sim 0.4$ for many pulse component pairs is in excellent agreement with the value derived by Cordes et al. (2004). They derive a value of 0.33 considering the giant pulses to be $100 \%$ polarised, amplitude modulated, scintillated shot noise. It also implies that these components have undergone similar scintillation effects, ruling out the possibility of any variation in the scattering medium on these timescales. The average correlation coefficients computed for the double pulses is consistent with the average value computed for the widely spaced pulse components (pulses in the top panel of Fig. 11). Since a clear roll-off in the values of correlation coefficient is not seen in the data presented here, we conclude that the scintillation timescales are longer than $14 \mathrm{~ms}$, which is entirely consistent with Cordes et al. (2004).

\section{Discussion}

To our knowledge this is the largest collection of high timeresolution giant pulse analysis presented in the literature. Even though some features of the giant pulse emission like the giant nano shots are in the process of being explained (Hankins \& Eilek 2007), several questions still remain about the pulsar 
emission mechanism in general and the giant pulse phenomena in particular. From the measured pulse widths and the observed structure in many pulses, it is evident from the analysis presented in this paper that the giant pulse emission is a manifestation of temporal plasma changes in the pulsar magnetosphere. The observed giant pulse rates are further evidence for this temporal variation, because if the mechanism responsible for the giant pulses is active on timescales longer than a pulse period, a clear excess of giant pulses separated by a single rotation period can be expected. On the basis of the giant pulse arrival times, it was concluded that the observed giant pulse emission does not come from a steady emission beam loosely bound to the stellar surface (Lundgren et al. 1995; Sallmen et al. 1999). We confirm that our data do not support such a model, for if such a beam with random wobbles operates, a characteristic width in the giant pulses can be expected. In other words, the distribution of the pulse widths would be normally distributed with a mean width.

The power-law nature of the giant pulse intensity distributions was shown by Lundgren et al. (1995), and they inferred that the normal pulses formed a separate part of the intensity distributions. In this work, we have shown conclusively that the giant pulses consist of two distinct populations especially for those pulses found at the inter pulse phase. We see a definite change in the shape of the distribution of pulse energies as we go to lower energies and we also see a slight broadening of the pulses. These pulses still seem to be distinct from what might be called "normal pulses": they are still narrower than most subpulses and are at least 27 times brighter than the normal pulses. The slope of the distribution containing these pulses is different from rest of the intensity distribution. These pulses could possibly be the trailing part of the distribution inferred by Lundgren et al. (1995). Moreover, how these relate to the precursor emission is unclear, which can clearly be improved upon using the double giant pulses. While there is evidence of a broadening of the pulses as they weaken in intensity, they do not appear to be as broad as standard subpulses. This finding has implications in the model derived by Petrova (2004), where a clear power-law distribution is explained, but not a weak giant population. The power-law index derived also has implications for interpreting giant pulse emission on the basis of self organised criticality (Bak et al. 1987), as suggested by Cairns (2004).

The spectral index of the Crab giant pulses reported in this work suggests that the emission bandwidth is at least $\Delta v / v>0.1$ and may approach the upper limit $\Delta v / v=0.2$ predicted in numerical models by Weatherall (1998). Hankins \& Eilek (2007) find a similar emission bandwidth at $9.5 \mathrm{GHz}$. Moreover, the average spectral index of giant pulses at the interpulse phase is flatter than the giant pulses at the main pulse phase. This possibly explains the dominant and bright nature of interpulse giants at $v>5 \mathrm{GHz}$. We note the prominent emergence of bimodality in the intensity distribution of the interpulses relative to the main phase pulses. Furthermore, (Hankins \& Eilek 2007) find upward drifting emission bands in the spectrum of the interpulses giants and not in the main pulse giants. These differences strongly suggest a different nature to the interpulses. To explain the drifting emission bands, Lyutikov (2007) derived an excess plasma density of $\sim 10^{5}$ and a large Lorentz factor of the emitting particles of the order of $\sim 10^{7}$, and this condition is satisfied close to the light cylinder over the magnetic equator. However, the model proposed by Lyutikov (2007) is only valid for $v>5 \mathrm{GHz}$, where the emission bands are observed. While results from our observations can neither support nor rule out this model, the difference in pulse intensity distributions we find indicates that the interpulse giants are different in nature.
It is worth noting that the pulsar signal is a stochastic process that contributes to the measurement noise of the pulsed intensity. This is especially true in the case of giant pulse emission, where pulsed flux can exceed $1500 \mathrm{Jy}$, an order of magnitude greater than the system equivalent flux density (SEFD) of approximately 145 Jy. Source-intrinsic noise increases the measurement uncertainty of various derived parameters, such as the pulsed flux density, pulse width, scattering time, and spectral index van Straten (2009). In addition, any temporal and/or spectral correlations either intrinsic to the giant pulse emission or induced by interstellar scintillation - will also affect the uncertainties of any derived parameters. The vast majority of the pulses presented in this analysis have average flux densities that are lower than the SEFD, and we do not expect that self-noise will significantly alter the results of this analysis. To accurately quantify the impact of self-noise on parameter distributions (such as those presented in Figs. 4, 5, 7, and 8) would require extensive simulations that are beyond the scope of the present work but may provide additional insight in a future paper.

The previously unreported double pulses we found are consistent with the occurrence rate on a purely probabilistic basis. Collecting even more of these pulse pairs would allow for better checks of the statistics of occurrence to ascertain that they are chance occurrences and not indicative of some longer term underlying phenomenon driving the giant pulse emisision. Moreover detecting more of these pulses at higher time resolution would provide further insight into the nature of these pulses. Hankins \& Eilek (2007) found that the giant pulses at the interpulse phase show an additional dispersion when compared to the pulses at the main pulse phase. The closest pulse pair they were able to examine were separated by $12 \mathrm{~min}$. One may gain new insight into the excess dispersion seen at the interpulse phase by examining the double giant pulses, which are the closest giant pulse pair possible.

Scattering analysis of single pulses presented in this paper show a variety of scattering times and corroborates with the analysis of Sallmen et al. (1999). They show that scattering from multiple screens or a single thick screen is excluded because of the observed frequency independence of the pulse component separation. From this it was concluded that the multiple components that make up the giant pulses are intrinisic to the emission mechanism. Using multiple components and the double pulses, we conclude that the scintillation timescales are greater than $14 \mathrm{~ms}$, which indicates that there are no large changes in the number density of the scattering medium along the line of sight through the nebula on similar timescales. That the multiple components we detect in the giant pulses are spaced by at least $25 \mu \mathrm{s}$ implies that the magnetosphere and/or the plasma does not change on these timescales, if the source intrinsic emission is less than $25 \mu \mathrm{s}$. On the other hand, giant pulses may consist of overlapping nano shots. In this case the competing models make use of plasma turbulence leading to modulational instablity (Weatherall 1998) or the induced Compton scattering of low-frequency radio waves (Petrova 2004) in the magnetosphere to explain the origin of the nano shots. While with our data we are not sensitive to the pulses less than $4.1 \mu$ s duration, there is an indication that the emission bandwidth $\Delta v / v>0.1$, suggesting that the pulses can potentially have structure as narrow as $3.6 \mathrm{~ns}$ at this frequency.

\section{Conclusions}

The large collection of single pulses we gathered has allowed us to perform a range of statistics with the data. After careful flux 
calibration, a detailed analysis of the pulse intensities, energies, widths, and separation times was done by computing distributions of these quantities. In the single-pulse intensity distributions, we find a clear evidence of two distinct populations in the giant pulses. The giant pulse separation times show a Poission distribution, and the rate of occurrence of giant pulses was determined. Spectral indices for a large number of giant pulses were computed with the narrowly spaced multi band data. Significant dispersion in the spectral indices was found and a small negative average spectral index was found for the main- and interpulse giants, and they are flatter than the average pulse emission. We also note that in some cases there is evidence for intensity modulation with bandwidths that are smaller than the full band but not consistent with scintillation effects. The previously undetected double giant pulses were presented and we find that they are not more frequent than would be expected by chance. The scatter time for a large number of giant pulses was determined by modelling the scatter broadening as an exponenial function and the distribution of scatter times was computed. The double giant pulses were reported for the first time and it is found that they are not very different from the normal giant pulses. Using multiple emission components either at the main- or interpulse phase and the double giant pulses, we find no evidence of variation of the scattering material on timescales shorter than $14 \mathrm{~ms}$ based on the correlation coefficient computed for emission within a single-pulse period.

Acknowledgements. The WSRT is operated by ASTRON. We thank the observers for setting up the observations. The PuMa-II instrument and one of us, R.K., are funded by Nederlands Onderzoekschool Voor Astronomie (NOVA). We acknowledge the use of SAO/NASA Astrophysics Data System. R.K. thanks Maciej Serylak for his helpful comments. We thank the anonymous referee for comments that improved this paper.

\section{References}

Argyle, E., \& Gower, J. F. R. 1972, ApJ, 175, L89

Backer, D. C., Wong, T., \& Valanju, J. 2000, ApJ, 543, 740

Bak, P., Tang, C., \& Wiesenfeld, K. 1987, Phys. Rev. Lett., 59, 381
Bhat, N. D. R., Wayth, R. B., Knight, H. S., et al. 2007, ApJ, 665, 618 Bhat, N. D. R., Tingay, S. J., \& Knight, H. S. 2008, ApJ, 676, 1200 Bietenholz, M. F., Kassim, N., Frail, D. A., et al. 1997, ApJ, 490, 291 Cairns, I. H. 2004, ApJ, 610, 948

Cairns, I. H., Johnston, S., \& Das, P. 2001, ApJ, 563, L65

Cooper, B. F. C. 1970, Aust. J. Phys., 23, 521

Cordes, J. M. 1986, ApJ, 311, 183

Cordes, J. M., \& Rickett, B. J. 1998, ApJ, 507, 846

Cordes, J. M., Bhat, N. D. R., Hankins, T. H., McLaughlin, M. A., \& Kern, J. 2004, ApJ, 612, 375

Dicke, R. H. 1946, Rev. Sci. Instrum., 17, 268

Eilek, J. A., Arendt, Jr., P. N., Hankins, T. H., \& Weatherall, J. C. 2002, in Neutron Stars, Pulsars, and Supernova Remnants, ed. W. Becker, H. Lesch, \& J. Trümper, 249

Gower, J. F. R., \& Argyle, E. 1972, ApJ, 171, L23

Hankins, T. H., \& Eilek, J. A. 2007, ApJ, 670, 693

Hankins, T. H., Kern, J. S., Weatherall, J. C., \& Eilek, J. A. 2003, Nature, 422, 141

Heiles, C., Campbell, D. B., \& Rankin, J. M. 1970, Nature, 226, 529

Hesse, K. H., \& Wielebinski, R. 1974, A\&A, 31, 409

Hotan, A. W., van Straten, W., \& Manchester, R. N. 2004, Proc. Astron. Soc. Aust., 21, 302

Johnston, S., van Straten, W., Kramer, M., \& Bailes, M. 2001, ApJ, 549, L101 Karuppusamy, R., Stappers, B., \& van Straten, W. 2008, PASP, 120, 191

Kuzmin, A., Losovsky, B. Y., Jordan, C. A., \& Smith, F. G. 2008, A\&A, 483, 13

Lorimer, D. R., \& Kramer, M. 2005, Handbook of Pulsar Astronomy (Cambridge University Press)

Lundgren, S. C., Cordes, J. M., Ulmer, M., et al. 1995, ApJ, 453, 433

Lyne, A. G., Pritchard, R. S., \& Smith, F. G. 1993, MNRAS, 265, 1003

Lyne, A. G., Pritchard, R. S., \& Graham-Smith, F. 2001, MNRAS, 321, 67

Lyubarskii, Y. E., \& Petrova, S. A. 1998, A\&A, 333, 181

Lyutikov, M. 2007, MNRAS, 381, 1190

Moffett, D. A., \& Hankins, T. H. 1994, BAAS, 184, 2103

Petrova, S. A. 2004, A\&A, 424, 227

Popov, M. V., \& Stappers, B. 2003, Astron. Rep., 47, 660

Popov, M. V., \& Stappers, B. 2007, A\&A, 470, 1003

Popov, M. V., Kuz'min, A. D., Ul'yanov, O. M., et al. 2006, Astron. Rep., 50, 562

Ritchings, R. T. 1976, MNRAS, 176, 249

Sallmen, S., Backer, D. C., Hankins, T. H., Moffett, D., \& Lundgren, S. 1999, ApJ, 517, 460

Staelin, D. H., \& Reifenstein, III, E. C. 1968, Science, 162, 1481

Staelin, D. H., \& Sutton, J. M. 1970, Nature, 226, 69

Taylor, J. H., \& Weisberg, J. M. 1989, ApJ, 345, 434

van Straten, W. 2009, ApJ, 694, 1413

Weatherall, J. C. 1998, ApJ, 506, 341

Williamson, I. P. 1972, MNRAS, 157, 55 